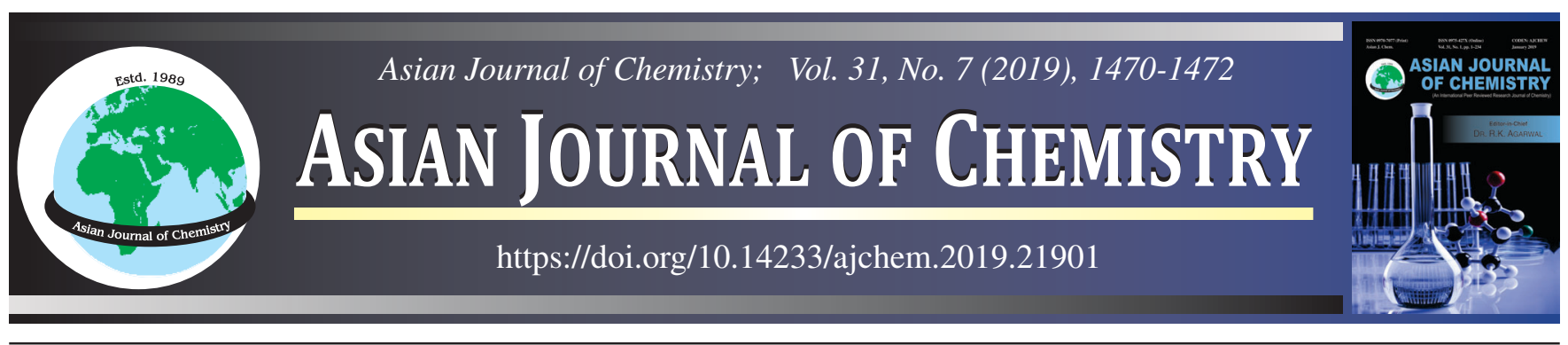

\title{
Knoevenagel Condensation Shadowed by Michael Addition \& O-Alkylation of Resorcinol, Malononitrile and Benzadehyde to form Pyrrolidine, Piperidine and Morpholine Substituted Benzopyran Derivatives in Dry $\mathrm{K}_{2} \mathrm{CO}_{3}$
}

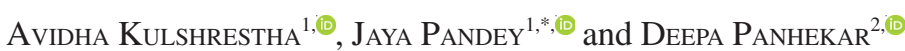

${ }^{1}$ Amity School of Applied Sciences, Amity University, Uttar Pradesh, Lucknow Campus, Lucknow-226010, India

${ }^{2}$ Department of Chemistry, Dr. Ambedkar College, Deeksha Bhoomi, Nagpur-440010, India

*Corresponding author: E-mail: jpandey@lko.amity.edu

Received: 28 December 2018;

Accepted: 12 February 2019;

Published online: 21 May 2019;

AJC-19398

\begin{abstract}
A condensation reaction of resorcinol, malononitrile and benzaldehyde in presence of sodium carbonate at room temperature on vigorous stirring gives 2-amino-7-hydroxy-4-phenyl-4H-1-benzopyran-3-carbonitrile which on alkylation in presence of dry potassium carbonate in dry acetone at $70-80^{\circ} \mathrm{C}$ with 1,2-chloroethyl pyrrolidine, 1,2-chloroethyl piperidine, 1,2-chloroethyl morpholine gives corresponding novel compounds.
\end{abstract}

Keywords: Knoevenagel condensation, Michael addition, 1,2-Chloroethyl pyrrolidine, Piperidine, Morpholine.

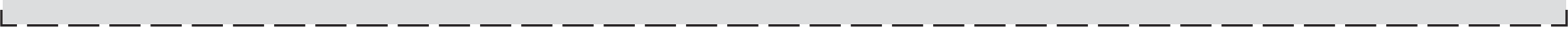

\section{INTRODUCTION}

It is well known that one pot multicomponent reactions are always better than multi-step synthesis, as they require minimal workup and desired product can be obtained in one pot often in quantitative yields [1,2]. Water is easily available solvent, use of water as solvent in the organic synthesis give rise to green chemistry. It is less pollutant, non-hazardous, non-combustible solvent these properties of water promote it in organic synthesis $[3,4]$. We report pyridine, piperidine and morpholine substituted 2-aminobenzopyran moiety which is very active and possess many biological activities. Formation of this moiety undergone Knoevenagel condensation, in which malononitrile (an active methylene compound) react with benzaldehyde in presence of tracing amount of methanol and aqueous solution of sodium carbonate and formed benzylidine propane dinitrile on loss of a water molecule. A Knoevenagel condensation is a nucleophilic addition of an active hydrogen compounds to a carbonyl group followed by dehydration reaction in which a molecule of water is eliminated [5,6]. Active hydrogen compounds viz. diethyl malonate, meldrum's acid, ethylacetoacetate, malonic acid, cyanoacetic acid, malononitrile, etc. [7-9].
In the same multi-component reaction second turn of the reaction undergone by Michael addition, in which benzyldine propanedinitrile reacts with 1,3-resorcinol and to form $[(2,4-$ dihydroxyphenyl)(phenyl)methyl]propane dinitrile which on cyclization and bond shifting formed 2-amino-7-hydroxy-4phenyl-4H-1-benzopyran-3-carbonitrile [10]. Michael addition is appropriate to the larger class of conjugated addition and the most useful method for $\mathrm{C}-\mathrm{C}$ bond formation. The Michael addition is thermodynamically controlled; the reaction donors are active methylene's such as malonates and nitroalkanes, and the acceptors are activated olefins such as $\alpha, \beta$-unsaturated carbonyl compounds $[11,12]$. The second step reaction undergone O-alkylation in which 1,2-chloroethylpyrrolidine,1,2chloroethylpiperidine or 1,2-chloroethylmorpholine act as alkylating agent and - $\mathrm{OH}$ part of substrate alkylate and given ether as product. The alkyl group may be transferred as an alkyl carbocation, a free radical, carbanion or a carbine or their equivalents $[13,14]$. When the alkylating agent is an alkyl halide, the conversion is known as Williamson ether synthesis. Alcohols are also considered as good alkylating agents in the presence of suitable acid/base catalysts [15].

The interest in benzopyrans and their derivatives increased since many years because these compounds are components

This is an open access journal, and articles are distributed under the terms of the Creative Commons Attribution-NonCommercial-ShareAlike 4.0 (CC BY-NC-SA 4.0) International License which allows readers to freely read, download, copy, distribute, print, search, or link to the full texts of its articles and to use them for any other lawful non-commercial purpose as long as the original source is duly acknowledged. 
of many naturally occurring products and have also been subjected to structural modifications for potential medicinal properties. 2-Amino-7-hydroxy-4-phenyl-4H-1-benzopyran-3carbonitrile (1a) had reported as in vitro cytotoxic activity on a human breast tumor cell line (MCF7, $\mathrm{IC}_{50} 8 \mathrm{nM}$ ) [16,17].

\section{EXPERIMENTAL}

Laboratory grade chemicals and solvents were purchased from Sigma Aldrich and Merck and used without any further purification. The reactions were monitored by TLC using Merck's silica gel 60F254 aluminium sheets. Infrared spectra were recorded neat on Agilent cary 630 spectrophotometer. High resolution mass spectra were recorded on Agilent 6520 (Q-TOF). NMR spectra were recorded on a Bruker Avance 400 (FT NMR) DPX300MHz NMR spectrometer.

Synthesis of 2-amino-7-hydroxy-4-phenyl-4H-1-benzopyran-3-carbonitrile (1a): A mixture of benzadehyde, malononitrile and resorcinol in 2:2:2 dissolved in $1 \mathrm{~mL}$ methanol in round bottom flask. A $20 \mathrm{~mL}$ solution of $10 \% \mathrm{Na}_{2} \mathrm{CO}_{3}$ was added in round bottom flask and the resulting suspension kept on vigorous stirring for $10 \mathrm{~h}$ at $25^{\circ} \mathrm{C}$. After the completion of reaction filter the solution and residue washed with water then cold methanol and dried in oven at $100{ }^{\circ} \mathrm{C}$ (Scheme-I).

2-Amino-4-phenyl-7-[2-(pyrrolidin-1-yl)ethoxy]-4H-1benzopyran-3-carbonitrile (1b): A mixture of 2-amino-3cyano-7-hydroxy-4-phenyl-4H-chromene (1a) $(0.264 \mathrm{~g}, 1$ $\mathrm{mmol}), 1$-(2-chloroethyl)pyrolidine $\cdot \mathrm{HCl}(0.255 \mathrm{~g}, 1.5 \mathrm{mmol})$ dry $\mathrm{K}_{2} \mathrm{CO}_{3}(0.276 \mathrm{~g} 2 \mathrm{mmol})$ dissolved in $25 \mathrm{~mL}$ dry acetone in $50 \mathrm{~mL}$ round bottom flask and refluxed the solution for $8 \mathrm{~h}$ at $60-70^{\circ} \mathrm{C}$. Cooled the solution at room temperature and workup with ethyl acetate and water, evaporate the solvent with rotatory evaporator and collected the yellow crystalline compound. Monitored the compound by TLC with methanol and chloroform (5:5).

2-Amino-4-phenyl-7-[2-(piperidin-1-yl)ethoxy]-4H-1benzopyran-3-carbonitrile (1c): A mixture of 2-amino-3cyano-7-hydroxy-4-phenyl-4H-chromene (1a) $(0.264 \mathrm{~g}, 1$ $\mathrm{mmol}), 1$-(2-chloroethyl)piperidine $\cdot \mathrm{HCl}(0.276 \mathrm{~g}, 1.5 \mathrm{mmol})$ dry $\mathrm{K}_{2} \mathrm{CO}_{3}(0.276 \mathrm{~g}, 2 \mathrm{mmol})$ dissolved in $25 \mathrm{~mL}$ dry acetone in $50 \mathrm{~mL}$ round bottom flask and refluxed the solution for $8 \mathrm{~h}$ at $60-70^{\circ} \mathrm{C}$. Cooled the solution at room temperature and workup with ethyl acetate and water, evaporate the solvent with rotatory evaporator and collected the bright pink crystalline compound. Monitored the compound by TLC with methanol and dichloromethane (DCM) $(4: 6)$
2-Amino-4-phenyl-7-[2-(morpholin-1-yl)ethoxy]-4H1-benzopyran-3-carbonitrile (1d): A mixture of 2-amino-3cyano-7-hydroxy-4-phenyl-4H-chromene (1a) $(0.264 \mathrm{~g}, 1$ mmol), 1-(2-chloroethyl)morpholine $\cdot \mathrm{HCl}(0.279 \mathrm{~g}, 1.5 \mathrm{mmol})$ dry $\mathrm{K}_{2} \mathrm{CO}_{3}(0.276 \mathrm{~g}, 2 \mathrm{mmol})$ dissolved in $25 \mathrm{~mL}$ dry acetone in $50 \mathrm{~mL}$ round bottom flask and then refluxed the solution for $8 \mathrm{~h}$ at $60-70{ }^{\circ} \mathrm{C}$. Cooled the solution at room temperature and workup with ethyl acetate and water, evaporate the solvent with rotatory evaporator and collect the yellow crystalline compound. Monitored the compound by TLC with methanol and dichloromethane (DCM) (4:6)

\section{Spectral data of selected compounds}

2-Amino-7-hydroxy-4-phenyl-4H-1-benzopyran-3carbonitrile (1a): Yield $85 \%$; white amorphous solid, m.p. $230-235^{\circ} \mathrm{C}$; IR ( KBr, $\left.v_{\max }, \mathrm{cm}^{-1}\right): 3495(\mathrm{R}-\mathrm{OH}), 3332\left(\mathrm{R}^{-\mathrm{NH}_{2}}\right)$, 2193 (R-CN), 1652 (C=CN vinyl nitrile), 1588-1406 (C-C arom.); ${ }^{1} \mathrm{H}$ NMR (400 MHz, DMSO $\delta$ ppm): 9.76 (s, Ar-OH ), 7.36-7.33 (t, $J=12 \mathrm{~Hz}), 7.26-7.20(\mathrm{q}, J=8 \mathrm{~Hz}) 6.90-6.84$ $(\mathrm{t}, J=12 \mathrm{~Hz}) 6.55-6.52(\mathrm{dd}, J=4 \mathrm{~Hz}), 6.478-6.472(\mathrm{~d}, J=$ $2.4 \mathrm{~Hz}), 4.66$ (s). ${ }^{13} \mathrm{C}$ NMR (100.60 MHz, DMSO $\left.\delta \mathrm{ppm}\right)$ : 160.23-160.20, 156.98, 148.82, 146.31, 129.88, 127.33, 126.62, 113.75, 112.34, (C-C arom.), 120.60 (CN), 102.14 (C=C vinylic), 56.28 (C-O). HRMS-EI ( $/ z / z)$ calcd. for $\mathrm{C}_{16} \mathrm{H}_{12} \mathrm{~N}_{2} \mathrm{O}_{2}: 264.2786$, found 264.2886. Elemental analysis \% calcd. (found): $\mathrm{C} 72.72$ (72.69), H 4.58 (4.60), N 10.60 (10.60).

2-Amino-4-phenyl-7-[2-(pyrrolidin-1-yl)ethoxy]-4H-1benzopyran-3-carbonitrile ( 1b): Yield $75 \%$; brown color solid, m.p. 265-272 ${ }^{\circ} \mathrm{C}$; IR $\left(\mathrm{KBr}, v_{\max }, \mathrm{cm}^{-1}\right)$ : $3334\left(\mathrm{R}-\mathrm{NH}_{2}\right)$, 2188 (R-CN), 1649 (-C=CN), 1579-1400 (C-C arom.),1336 ($\mathrm{C}-\mathrm{OR}, \mathrm{OR}=$ ethoxypyrrolidine $) ;{ }^{1} \mathrm{H} \mathrm{NMR}\left(300 \mathrm{MHz}, \mathrm{CDCl}_{3}\right.$ $\delta \mathrm{ppm}): 7.24-7.23(\mathrm{t}, J=1.2 \mathrm{~Hz}), 7.21-7.20(\mathrm{~d}, J=1.5 \mathrm{~Hz})$, $7.18(\mathrm{~s}), 7.15-7.14(\mathrm{t}, J=1.35), 7.13-7.12(\mathrm{t}, J=5.4), 7.105-$ $7.100(\mathrm{~d}, J=1.5 \mathrm{~Hz}), 6.76(\mathrm{~s}), 6.73(\mathrm{~s}), 6.54-6.53(\mathrm{~d}, J=2.4$ $\mathrm{Hz}), 6.519-6.510(\mathrm{~d}, J=2.7 \mathrm{~Hz}), 6.47-6.46(\mathrm{~d}, J=2.7 \mathrm{~Hz})$, $4.18(\mathrm{~s}), 4.56(\mathrm{~s}), 4.00-3.96(\mathrm{t}, J=11.7 \mathrm{~Hz}), 2.83-2.79(\mathrm{t}, J=$ $11.7 \mathrm{~Hz}), 2.57-2.53(\mathrm{t}, J=12.9 \mathrm{~Hz}), 1.96(\mathrm{~s}), 1.89(\mathrm{~s}), 1.77-$ $1.68(\mathrm{~m}, J=7.5 \mathrm{~Hz}) .{ }^{13} \mathrm{C}$ NMR $\left(75.49 \mathrm{MHz}, \mathrm{CDCl}_{3}, \delta \mathrm{ppm}\right)$ : $159.50,158.67,149.31,145.06,130.34,129.31,128.90,128.02$, $127.29,120.26,115.16,112.25,102.13,77.65,77.23,67.29$, $60.67,59.29,54.96,54.72,40.61,23.60,1.16$. HRMS-EI $(\mathrm{m} / \mathrm{z})$ calcd. for $\mathrm{C}_{16} \mathrm{H}_{12} \mathrm{~N}_{2} \mathrm{O}_{3}$ : 361.43692; ESI-SCAN-MS: + 362.18. Elemental analysis \% calcd. (found): C 73.11 (73.15), H 6.41 (6.45), N 8.16 (8.16).

2-Amino-4-phenyl-7-[2-(piperidin-1-yl)ethoxy]-4H-1benzopyran-3-carbonitrile (1c): Yield $70 \%$; bright pink color<smiles>[R]c1ccc(C2C(C#N)=C(N)Oc3cc(O)ccc32)cc1[3H]</smiles><smiles>[R7]CCOc1ccc2c(c1)OC(N)=C(C#N)C2c1ccc([R])c([R])c1</smiles>

Where $\mathrm{R}=\mathrm{H}, \mathrm{Br}, \mathrm{OCH}_{3}, \mathrm{OH} \quad \mathrm{R}^{1}=\mathrm{H}, \mathrm{OH}, \mathrm{OCH}_{3} \mathrm{R}^{2}=$ Pyrrolidine, Piperidine, Morpholine 
solid, m.p. $275-280^{\circ} \mathrm{C}$; IR $\left(\mathrm{KBr}, v_{\max }, \mathrm{cm}^{-1}\right): 3350\left(\mathrm{R}-\mathrm{NH}_{2}\right), 2240$ (R-CN), 1639 (C=CN vinyl nitrile), 1589-1450 (C-C arom.), 1302 (C-OR, OR = ethoxypiperidine); ${ }^{1} \mathrm{H}$ NMR $(300 \mathrm{MHz}$, $\left.\mathrm{CDCl}_{3} \delta \mathrm{ppm}\right): 7.26-7.24(\mathrm{t}, J=3 \mathrm{~Hz}), 7.214-7.209(\mathrm{~d}=1.5)$, 7.189 (s), 7.15-7.14 (d, $J=3 \mathrm{~Hz}), 6.77$ (s), 6.75 (s), 6.547$6.539(\mathrm{~d}, J=2.4 \mathrm{~Hz}), 6.519-6.510(\mathrm{~d}, J=2.7 \mathrm{~Hz}), 6.472-6.462$ (d, $J=2.7 \mathrm{~Hz}), 4.819$ (s), 4.565 (s), 4.009-3.966 (t, $J=6.9$ Hz), 2.98 (s) 2.833-2.794 (t, $J=5.7 \mathrm{~Hz}$ ), 2.576-2.533 (q, $J=$ $6.45 \mathrm{~Hz}), 1.96$ (s), 1.89 (s). ${ }^{13} \mathrm{C} \mathrm{NMR}\left(75.49 \mathrm{MHz}, \mathrm{CDCl}_{3}, \delta\right.$ ppm): 157.31, 155.55, 155.20, 131.06, 129.33, 129.10, 119.99, 115.16, 114.25, 104.25, 77.44, 77.23, 62.88, 61.86, 57.88, 55.09, 42.22, 34.55, 31.96. HRMS-EI $(\mathrm{m} / \mathrm{z})$ calcd. for $\mathrm{C}_{23} \mathrm{H}_{25} \mathrm{~N}_{3} \mathrm{O}_{2}$ : 375.4635; ESI-SCAN-MS: +376.09. Elemental analysis \% calcd. (found): C 71.79 (71.83), H 6.43 (6.44), N 11.16 (11.19).

2-Amino-4-phenyl-7-[2-(morpholin-1-yl)ethoxy]-4H1-benzopyran-3-carbonitrile (1d): Yield $68 \%$; yellow color solid; m.p. $275-280{ }^{\circ} \mathrm{C}$; IR $\left(\mathrm{KBr}, v_{\max }, \mathrm{cm}^{-1}\right): 3335\left(\mathrm{R}-\mathrm{NH}_{2}\right)$, 2198 ( $\mathrm{R}-\mathrm{CN}$ ), 1644 ( $\mathrm{C}=\mathrm{CN}$ vinyl nitrile), 1599-1442 (C-C arom.), 1335 (C-OR, OR = ethoxymorpholine); ${ }^{1} \mathrm{H}$ NMR $(300 \mathrm{MHz}$, $\mathrm{CDCl}_{3} \delta \mathrm{ppm}$ ): 7.255-7.241 (t, $\left.J=4.2 \mathrm{~Hz}\right), 7.112-7.090(\mathrm{~d}, J$ $=6.6 \mathrm{~Hz}), 7.009(\mathrm{~s}), 6.990-6.987(\mathrm{~d}, J=0.9 \mathrm{~Hz}), 6.88(\mathrm{~s})$, 6.76 (s), 6.455-6.454 (d, $J=0.3 \mathrm{~Hz}), 6.424-6.420$ (d, $J=1.2$ $\mathrm{Hz}), 4.908(\mathrm{~s}), 4.670(\mathrm{~s}), 4.098-4.086$ ( t $J=3.6 \mathrm{~Hz}), 2.992$ (s), 2.883-2.862 (t, $J=6.3 \mathrm{~Hz}), 2.588-2.537(\mathrm{q}, J=7.65 \mathrm{~Hz}$ ), 2.003 (s), 1.962 (s), 1.884 (s). ${ }^{13} \mathrm{C} \mathrm{NMR}\left(75.49 \mathrm{MHz}, \mathrm{CDCl}_{3}, \delta\right.$ ppm): 159.66, 156.31, 153.55, 152.20, 135.96, 129.33, 129.10, $120.99,115.16,114.85,104.25,77.98,77.23,65.88,61.86$, 57.88, 55.70, 42.22, 39.33, 35.65, 31.22. HRMS-EI $(\mathrm{m} / \mathrm{z})$ calcd. for $\mathrm{C}_{22} \mathrm{H}_{23} \mathrm{~N}_{3} \mathrm{O}_{3}$ : 377.43632; ESI-SCAN-MS: +377.47509. Elemental analysis \% calcd. (found): C 70.01 (70.83), H 6.14 (6.22), N 11.13 (11.15).

2-Amino-4-(3-bromophenyl)-7-hydroxy-4H-1-benzopyran-3-carbonitrile (2a): Yield $80 \%$; off white color solid; m.p. $265-270^{\circ} \mathrm{C}$; IR $\left(\mathrm{KBr}, v_{\max }, \mathrm{cm}^{-1}\right): 3443(\mathrm{R}-\mathrm{OH}), 3335$ (R$\left.\mathrm{NH}_{2}\right), 2192$ (R-CN), $1640(\mathrm{C}=\mathrm{CN}$, vinylnitrile), 1587-1410, (C-C arom.), 720 (R-C-Br); ${ }^{1} \mathrm{H}$ NMR (400 MHz, DMSO $\delta$ ppm): 8.35 (s), 7.47-7.39 (t, $J=22 \mathrm{~Hz}), 7.35-7.31(\mathrm{t}, J=16$ $\mathrm{Hz}), 7.24-7.22(\mathrm{~d}, J=8 \mathrm{~Hz}), 6.99$ (s), 6.88-6.86 (d, $J=8 \mathrm{~Hz})$, 6.577-6.480 (ddd, $J=2.4 \mathrm{~Hz}), 4.73$ (s). ${ }^{13} \mathrm{C}$ NMR $(100.60 \mathrm{MHz}$, DMSO $\delta$ ppm): 160.34, 160.30, 157.20, 149.06, 148.78, 130.89, 129.94, 129.88, 129.58, 126.55, 12181, 120.412, 112.97, $112.51,102.24,55.65$. HRMS-EI $(\mathrm{m} / \mathrm{z})$ calcd. for $\mathrm{C}_{16} \mathrm{H}_{11} \mathrm{~N}_{2} \mathrm{O}_{2} \mathrm{Br}, 343.1747$, found 343.1751. Elemental analysis $\%$ calcd. (found): C 56.00 (56.11), H 3.23 (3.26), N 8.16 (8.15).

\section{RESULTS AND DISCUSSION}

In the present work, a multi component reaction in which active methylene compounds (malononitrile) reacted with carbonyl compounds (benzaldehyde and substituted benzaldehyde) in presence of tracing amount of methanol and weak basic medium (aq. $\mathrm{Na}_{2} \mathrm{CO}_{3}$ ) and formed benzylidine propanedinitrile. The benzylidinepropanedinitrile reacted with resorcinol and formed [(2,4-dihydroxyphenyl)(phenyl)methyl]propanedinitrile which on cyclization given 7-hydroxy-2-imino-4-phenyl3,4-dihydro- $2 H$-1-benzopyran-3-carbonitrile by hydrogen shift it converted into 2-amino-7-hydroxy-4-phenyl-4H-1benzopyran-3-carbonitrile. In the second step, above formed compounds reacted with pyrrlidine, piperidine and morpholine substituted alkyl halide, in presence of dry. $\mathrm{K}_{2} \mathrm{CO}_{3}$ and dry acetone as medium to form novel benzopyran derivatives. Simple reaction conditions and easy available catalyst made it favourable for synthesis in high yields.

\section{ACKNOWLEDGEMENTS}

The authors are thankful for financial support from DST (Grant registration \# CS-176/2013) and UPCST (Grant registration\# CST/D 6547/2017) and Amity University, Uttar Pradesh, Lucknow Campus, India for providing the research facilities.

\section{CONFLICT OF INTEREST}

The authors declare that there is no conflict of interests regarding the publication of this article.

\section{REFERENCES}

1. D. Andreou, M.G. Kallitsakis, E. Loukopoulos, C. Gabriel, G.E. Kostakis and I.N. Lykakis, J. Org. Chem., 83, 2104 (2018); https://doi.org/10.1021/acs.joc.7b03051.

2. X.W. Zhang, W.L. Hu, S. Chen and X.G. Hu, Org. Lett., 20, 860 (2018); https://doi.org/10.1021/acs.orglett.7b04028.

3. A. G. Neo and C. F. Marcos, Org. Lett., 20, 3875 (2018); https://doi.org/10.1021/acs.orglett.8b01462.

4. N.R. Candeias, P.M.S.D. Cal, V. André, M.T. Duarte, L.F. Veiros and P.M.P. Gois, Tetrahedron, 66, 2736 (2010); https://doi.org/10.1016/j.tet.2010.01.084.

5. I.B. Masesane and S.O. Mihigo, Synth. Commun., 45, 1546 (2015); https://doi.org/10.1080/00397911.2015.1031249.

6. Y. Ogiwara, K. Takahashi, T. Kitazawa and N. Sakai, J. Org. Chem., 80, 3101 (2015); https://doi.org/10.1021/acs.joc.5b00011.

7. M. Pourmohammad and M. Mokhtary, C.R. Chim., 18, 554 (2015); https://doi.org/10.1016/j.crci.2014.09.008.

8. M.R. Naimi-Jamal, S. Mashkouri and A. Sharifi, Mol. Divers., 14, 473 (2010);

https://doi.org/10.1007/s11030-010-9246-5.

9. J. Albadi, A. Razeghi, A. Mansournezhad and Z. Azarian, J. Nanostr. Chem., 3, 85 (2013);

https://doi.org/10.1186/2193-8865-3-85.

10. P. Wadhwa, A. Kharbanda and A. Sharma, Asian J. Org. Chem., 7, 634 (2018); https://doi.org/10.1002/ajoc.201700609.

11. S. Lauzon, M. Li, H. Keipour and T. Ollevier, Eur. J. Org. Chem., 2018, 4536 (2018); https://doi.org/10.1002/ejoc.201800780.

12. R. Singh, Y. Thopate, D. Equbal and A.K. Sinha, Adv. Synth. Catal., 360, 4412 (2018); https://doi.org/10.1002/adsc.201801150.

13. Y. Zuo, N. Yang, X. Huang, C. Hu and Z. Su, J. Org. Chem., 83, 4628 (2018); https://doi.org/10.1021/acs.joc.8b00387.

14. Y.Z. Hua, J.W. Chen, H. Yang and M.C. Wang, J. Org. Chem., 83, 1160 (2018); https://doi.org/10.1021/acs.joc.7b02599.

15. H.R. Safaei, M. Shekouhy, S. Rahmanpur and A. Shirinfeshan, Green Chem., 14, 1696 (2012); https://doi.org/10.1039/c2gc35135h.

16. M.M. Kandeel, A.M. Kamal, E.K.A. Abdelall, H.A.H. Elshemy, Der Pharm. Chem., 4, 1653 (2012).

17. A. Bouattour, M. Fakhfakh, S. Abid, L. Paquin, R.L. Guvel, A. Corlu, S. Ruchaud, S. Bach, H. Ammar and J.-P. Bazureau, ARKIVOC, 291 (2017); https://doi.org/10.24820/ark.5550190.p010.040. 